But can these bacteria be used to treat human cancers? Future experiments are required to answer safety questions, as the rapid destruction of large tumours was toxic and caused the death of some animals. It will also be important to determine the mechanism by which C. novyi selectively destroys viable tumour cells that are adjacent to hypoxic areas. The authors also point out that not all tumours will be susceptible to COBALT, and they will have to determine which chemotherapeutic drugs act synergistically with bacteria against human tumours.

Kristine Novak

(D) References and links ORIGINAL RESEARCH PAPER Dang, L. H. Bettegowda, C., Husa, D. L., Kinzler, K. W. \& Vogelstein, B. Combination bacteriolytic therapy for the treatment of experimental tumors. Proc. Natl Acad. Sci. (2001) Nov 27; [epub ahead of print]

FURTHER READING Sznol, M. et al. Use of preferentially replicating bacteria for the treatment of cancer. J. Clin. Invest. 105, 1027-1030 (2000) WEB SITE

Bert Vogelstein's lab: http://www.med.jhu.edu/ pharmacology/pages/faculty/vogelstein.html

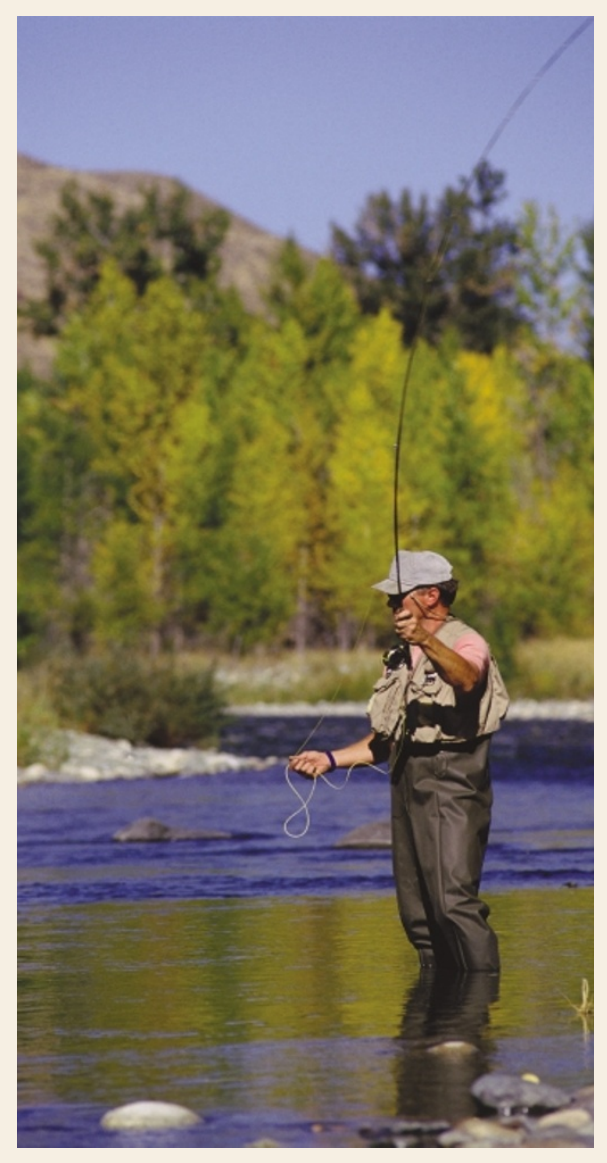

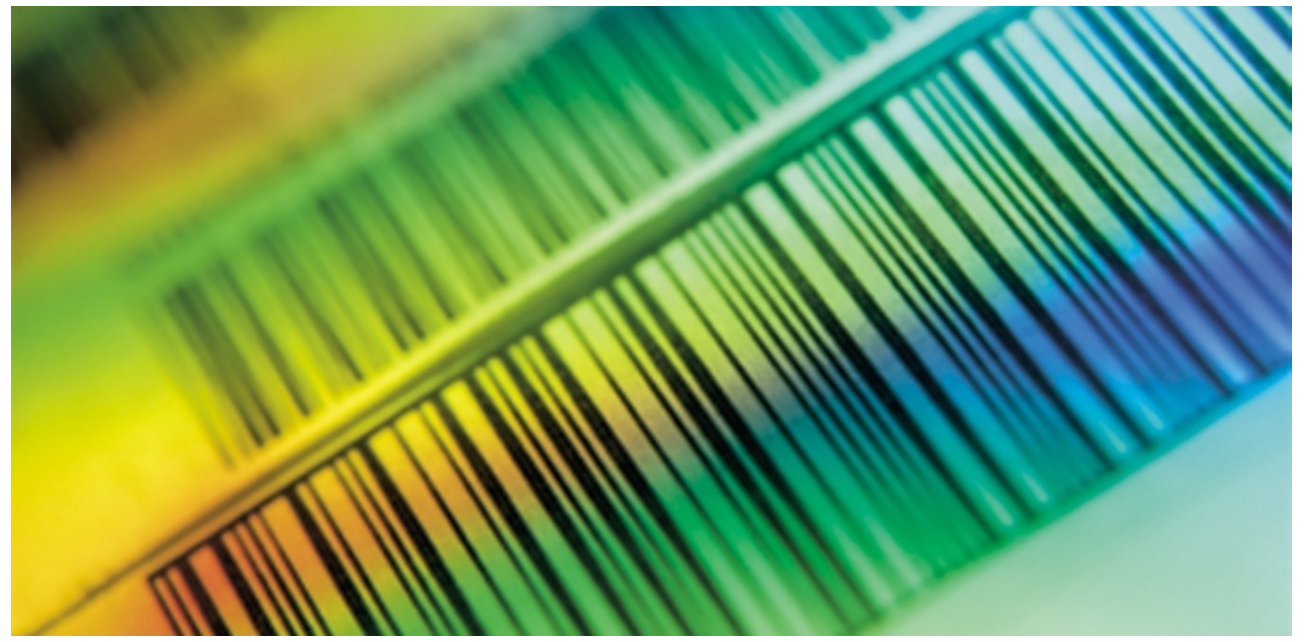

GENOMIC INSTABILITY

\title{
Protecting the code
}

A code of post-translational modifications including methylation, acetylation and phosphorylation - in the amino termini of histones determines the level of chromatin compaction. Histone acetylation is now recognized as an important therapeutic target in cancer, but what about other histone modifications? Thomas Jenuwein's group now provides the first indication that histone methylation might also influence tumour formation, by regulating genome stability.

Previous studies have indicated that the Suv39h enzymes - which methylate histone $\mathrm{H} 3$ at Lys9 - are required for correct chromosome segregation, so the authors generated knockout mice to investigate this function. Mice have two Suv39h genes - Suv39h1 and Suv39h2. Both are expressed during embryogenesis, and Suv39h2 is expressed in the testes of adult mice. Mice deficient in either Suv39h1 or Suv39h2 develop normally, presumably because the proteins act redundantly during embryonic development, but only a third of the expected number of double mutant mice (Suv39h dn) were born. Those that survived to birth were growth retarded and the males had underdeveloped testes. But are the Suv39h proteins required for chromosome segregation? A DNA content analysis of passaged primary mouse embryo fibroblasts (PMEFs) isolated from wild-type and Suv39h dn fetuses indicates that they are. After eight passages, wild-type PMEFs have largely senesced, but Suv39h dn PMEFs continue to proliferate and some have a polyploid DNA content. Interestingly, the chromosome morphology seems normal, indicating that whole chromosome sets are missegregated.

So, does the genomic instability induced in these mice increase susceptibility to tumour formation? Late-onset B-cell lymphomas which most closely resembled slowly progressing non-Hodgkin's lymphomas in humans developed in $33 \%$ of Suv $39 \mathrm{~h}$ dn mice, compared with none of the wild-type mice. Karotypic analysis of the lymphomas revealed that some had 'butterfly' chromosomes, which did not segregate as they remained attached at their centromeric regions. Could this be due to an effect on the pericentric heterochromatin region in these cells?

The authors used an antibody that acts as a marker of heterochromatin - by specifically recognizing $\mathrm{H} 3$ methylated at Lys9 - to show that both Lys9 methylation and heterochromatin were absent in Suv39h dn cells. But could modification of other histone residues that are known to be important for pericentric heterochromatin organization also be altered by lack of Lys9 methylation? A combination of immunoblotting and immunofluorescence revealed that phosphorylation of Ser10 in H3, and acetylation of Lys9 of $\mathrm{H} 3$ and Lys12 of H4, are affected by loss of Suv39h activity.

As mentioned, Suv39h2 is also expressed in the testes, and Suv39h dn males have impaired spermatogenesis, characterized by the delayed onset of meiotic prophase and a subsequent increase in apoptosis. Interestingly, chromosome spreads show that there are many nonhomologous interactions between chromosomes, predominantly between centromeres. This indicates a mechansim by which genomic stability is impaired in Suv39h-deficient mice.

So, the Suv39h methyltransferases comprise a potential new class of tumour suppressors that maintain genome stability by ensuring that the histone code is preserved in pericentric heterochromatin. The hunt is now on for loss of Suv39h function in human tumours.

Emma Greenwood

\section{(2) References and links}

ORIGINAL RESEARCH PAPER Peters, A. H. F. M. et al. Loss of the Sur39h histone methyltransferases impairs mammalian heterochromatin and genome stability. Cell 107, 323-337 (2001)

WEB SITE

Thomas Jenuwein's lab:

www.imp.univie.ac.at/groups/res.html/jenuwein 